\title{
Exploratory Geovisualizations for Supporting the Qualitative Analysis and Synthesis of Place-Related Emotion Data
}

Locations become places through personal significance and experience. While place data are not emotion data, per se, personal significance and experience are often emotional. In this paper, we explore the potential of using visual data exploration to support the qualitative analysis of place-related emotion data. To do so, we draw upon Creswell's (2009) definition of place to define a generic data model that contains emotion data for a given location and its locale. For each data dimension in our model, we present symbolization options that can be combined to create a range of interactive visualizations, specifically supporting re-expression. We discuss the usefulness of example visualizations, created based on a data set from a pilot study on how elderly women experience their neighborhood. We find that the visualizations support four broad qualitative data analysis tasks: revising categorizations, making connections and relationships, aggregating for synthesis, and corroborating evidence by combining sense of place with locale information to support a holistic interpretation of place data. In conclusion, the paper contributes to the literature in three ways. It provides a generic data model and associated symbolization options, and uses examples to show how place-related emotion data can be visualized. Further, the example visualizations make explicit how re-expression, the combination of emotion data with locale information, and visualization of vagueness and linked data support the analysis of emotion data. Finally, we advocate for visualization-supported qualitative data analysis in interdisciplinary teams so that more suitable maps are used and so that cartographers can better understand and support qualitative data analysis.

KEYWORDS: emotion data; place; visual data exploration; qualitative data analysis; QDA, qualitative analysis tasks; interdisciplinary, re-expression; geovisualization

\section{INTRODUCTION}

\subsection{MOTIVATION AND BACKGROUND}

People live in places, which are "locations imbued with meaning" and "sites of everyday practice" (Cresswell 2009, 9). These are not new concepts: at least as far back as the Greek philosophers, we have been thinking about places and our relationships with them. Tuan (1979) defined the concept of place as a space endowed with meaning, in which experience is a central notion. Places are not only located somewhere on Earth, but they are also connected to personal or shared significance, experience, emotion, or understanding (Lengen 2016). While people live in and interact with places, those places themselves also are important for people's wellbeing (Gebhard and Kistemann 2016), which is another dimension connected to emotions (e.g., Berrios et al. 2018).
In a range of application areas, analyzing the thoughts, emotions, and relationships associated with locations has been important, for example, both for understanding the quality of urban spaces and for transforming them. Examples of such analyses include studying pedestrian friendliness (Ferreira et al. 2016), judging the importance of reported city maintenance issues (Masdeval and Veloso 2015), or learning about the effect of greenness of places on wellbeing (Han 2017). In another specific example, elderly women reported that their ability (or inability) to engage in physical activities related to places (such as dancing, gardening, or walking) was the source of a range of emotions from fear and frustration to satisfaction and pleasure (Bennett et al. 2017). To form the basis of such 
analyses, different methods can be used to capture data on the relationship between places and emotions. In our own project, discussed below, we made use of data collected from "walking interviews," in which informants are accompanied and asked questions as they move through an area. Jones et al. point out that this method "has great potential to shed light on how participants use and understand different spaces" $(2008,7)$.

Once data have been collected about the spatial relationships between places and emotions, it may seem obvious for cartographers to map them. However, Jones et al. (2008) list a surprising number of projects that made little or no use of maps for exploring data captured through walking interviews. Furthermore, mapping poses some challenges. We tend naturally to believe that what is presented on a map is the reality (Wood 1992), but all mapped data have some degree of uncertainty, as is found in any knowledge production process (Couclelis 2003). The challenge of representing the uncertainty or vagueness associated with data has been an active research area (e.g., MacEachren 1992; Kinkeldey et al. 2017; Smith Mason et al. 2016). The difficulty lies not only in finding a suitable technique for displaying uncertainty, but also, or maybe even more so, in communicating its variability or ultimately assigning it a value or a quality that supports the purpose of the visualization. Additionally, maps often only depict snapshots of specific times (Barclay 2013). However, technological advances have now made it possible to create interactive visualizations of temporal data. While doing so remains challenging (Çöltekin et al. 2017), depicting temporal data can be useful for understanding changes over time. Time and the analysis of changes over time is important with respect to emotion data, as emotions may be related to memories and evolve over time (Jager 2016).

Traditionally, cartographers have paid little attention to the mapping of emotions. Griffin and McQuoid (2012) give an overview of how emotions are represented on maps and observe that emotion, contrary to the inter-related topic of cognition, has received little attention in the cartographic literature. With new data sources (e.g., from directed or undirected online activities), sentiment or emotion analysis has become a research topic in many areas (e.g., urban issues: Masdeval and Veloso 2015), but uses surprisingly few maps. The Literature Atlas - a project which aims to map the geographies of fiction-does not specifically map emotions, but deals with similar challenges in localizing and symbolizing information (Piatti and Hurni 2009; Piatti, Reuschel, and Hurni 2013).

\subsection{GOALS}

The work we report here is intended to support researchers analyzing place-related emotion data, by offering them exploratory geovisualizations that are able to include all relevant aspects of the data: topic, space, and time. To do so, we defined, from a geovisualization perspective, and refined, from a social science perspective, a generic data model as well as a set of multivariate interactive symbolization options for representing the dimensions of the data model. We created visualizations based on the symbolization options with the aim of supporting and enhancing the different stages of qualitative data analysis (QDA) from data description to synthesis and sensemaking. To create example visualizations, we employed a data set from a pilot study that aimed to understand the different dimensions of walkability and the social interaction potential of the neighborhoods of elderly women (Bachmann et al. 2016; Süsstrunk et al. 2018). However, we based our exploration of visualization options on a generic data model, its associated symbolization options, and general stages and principles of data analysis, and only exemplify the visualization options with the data from the case study. We present the examples to support understanding of the transferability of the described visualization approaches options to other application areas and data sets that contain place-related emotion data.

\section{EMOTIONS AND PLACES}

\subsection{EMOTIONS AND THEIR REFLECTIONS IN EXPRESSIONS}

EMOTIONS ARE IMPORTANT PARTS of all our lives, and we can even become emotional when talking about emotion (Coppin and Sander 2016). Still, we have difficulties giving a definition for the concept of emotion (Fehr and Russell 1984). Acknowledging the difficulty of defining the concept of emotion, Ekman (1984) proposed ten characteristics that allow distinguishing emotions from moods, reflexes, or attitudes. Studies confirmed that six basic emotions (fear, anger, surprise, disgust, sadness, and happiness) are experienced across cultures and can be conveyed through facial expressions when not suppressed or changed through social display rules (Ekman 1984). 
Empirical data show that emotion can impact human cognition and behavior, for example, by guiding attention, memory, decision-making, and action (Coppin and Sander 2016).

Recent research has used detailed categorizations to measure emotions through self-reporting (e.g., Kremer and den Uijl 2016). Importantly, emotions are not static, but evolve continuously (Kuppens and Verduyn 2017). The older people become, the more they shift their focus to more positive experiences and emotions (Charles and Leger 2016; Kremer and den Uijl 2016). Measuring explicit emotions, (i.e., conscious feelings), is challenging in itself, but especially with older persons, who are more heterogeneous in their personal characteristics than the young (Moschis 2003; Hayden et al. 2011), and who potentially experience various forms of decline that limit their abilities to focus and to express themselves (Kremer and den Uij1 2016). Explicit measurement tools, such as rating scales, may miss unconscious or implicit emotions. To capture implicit emotions, indirect approaches are required, such as text or sentiment analysis (Kremer and den Uijl 2016).

Sentiment analysis is an active research area in natural language processing. Merriam-Webster.com defines sentiment as "an attitude, thought, or judgment prompted by feeling" or "the emotional significance of a passage or expression as distinguished from its verbal context." Most commonly, sentiment analysis refers to the evaluation of the valence (i.e., positive, negative, or neutral) of statements or other pieces of text (Mohammad 2016). In addition to the categorization of valence, more complex descriptions and categorizations of sentiment with respect to different purposes such as market research exist. However, those are, with few exceptions, often developed by companies and organizations and are not publicly available (Iglesias et al. 2017).

\subsection{EMOTIONS AND THEIR RELATION TO LOCATIONS}

As we mentioned in our introduction, a person's environment is not just a specific part of the Earth's surface, but rather a multitude of places of significance and importance to the person. Cresswell $(2009,1)$ usefully summarized earlier conceptualizations of space and place (e.g., Tuan 1979) to define place as being tripartite.

The word "place" has been ... conceptualized as a particular location that has acquired a set of meanings and attachments. Place is a meaningful site that combines locations, locale, and sense of place. Location refers to an absolute point in space with a specific set of coordinates and measurable distances from other locations. Location refers to the "where" of place. Locale refers to the material setting for social relations-the way a place looks. Locale includes the buildings, streets, parks, and other visible and tangible aspects of a place. Sense of place refers to the more nebulous meanings associated with a place: the feelings and emotions a place evokes. These meanings can be individual and based on personal biography or they can be shared. Shared senses of place are based on mediation and representation. (Cresswell, 2009, 1)

Cresswell's definition allows combining people's expressions and emotions (the sense of place) with a specific locale (the available infrastructure at a location that can be defined through coordinates). This definition supports the combination of subjective and objective data at a location. Any framework for representing and analyzing places needs to account for its multiplicity. Additionally, unless a shared sense of place is the focus, the sense of place varies from person to person and it can, in any case, change over time and depending on the context. Therefore, sense of place categorizations are typically non-comprehensive but focused on specific aspects that are relevant within a certain context. For example, Deutsch, Yoon, and Goulias (2013) derived six categories of sense of place from surveys to explain travel behavior: attachment, association, identity, satisfaction, atmosphere, and community. Jenkins et al. (2016) tried to characterize locations through emerging themes. They aimed to derive a shared sense of place from crowd-sourced data using the categories of politics, sports, recreation, education, entertainment, and business.

\section{CASE STUDY FOR EXAMPLE VISUALIZATIONS}

The MABF (Mit Den Augen Betagter Frauen: "With the Eyes of Elderly Women") pilot study (Bachmann et al. 2016; Süsstrunk et al. 2018) developed an age- and gender-relevant method for capturing, analyzing, and 
discussing the physical and social environment of elderly women. For data collection, the study employed the "goalong" variant (Carpiano 2009) of a walking interview (Jones et al. 2008). Social scientists accompanied seven elderly women on a route through their daily routines to learn about different dimensions of walkability and the social interaction potential of the neighborhoods that they use actively. Audio recordings were made of the women's comments during the route, and were analyzed using qualitative content analysis (Bachmann et al. 2016). The results were compared to a GIS-based quantitative analysis of the locale such as footpath slope, numbers of benches for resting, and density of street lighting. This information was used to derive and visualize different walkability indices (Hollenstein and Bleisch 2016; Bleisch and Hollenstein 2017). The pilot study demonstrated the utility of the implemented research methods and identified important factors that support daily activities, independence, and social integration of elderly women in their living spaces.

The data collected and analyzed in the MABF pilot study, especially the statements that included emotional content like memories and personal experiences of a locale, were used to explore visualization options, below, that could support qualitative data analysis tasks for relating emotions and places. The social scientists who designed and led the MABF study were involved in discussing the visualization options and provided their perspective and general data analysis knowledge for focusing and refining the data model, the symbolization, and the visualizations proposed here. Below, the case study data are described in as much detail as is needed to understand the visualization methods and examples presented in this paper. However, during the MABF study, the data were not analyzed using all the methods proposed, nor do we comprehensively (re-) analyze the data, nor do we draw social scientific conclusions based on the examples in this paper.

\subsection{APPROXIMATING A SENSE OF PLACE}

Using Cresswell's (2009) tripartite place definition, we reconsidered the MABF data. All the data were related to the space the elderly women used, thus, they had a $10-$ cation. The GIS-based walkability analysis described the locale of each place, for example the density of lighting posts along a street. In the QDA portion of the study, the women's comments (for example, about their experience of the lighting: avoiding a street at night for its gloominess) were categorized in two dimensions: by their valence, and by their sense of place-the participants' relationship to or experience of aspects of the locale (Bachmann et al. 2016). The entire categorization was derived from an inductive-deductive process (Mayring 2014) based on existing literature and based on the walking interview data itself.

Seven sense of place categories resulted from this process:

- aesthetics (e.g., greenness, cleanliness, including aspects of wellbeing),

- walking comfort (e.g., experience of slope of pedestrian walkways),

- safety (e.g., experience of lighting, pedestrian crossings),

- facilities (e.g., relevance of shopping, restaurants),

- accessibility (e.g., experience of public transport options, judged distances to facilities),

- interaction (e.g., social contacts, comfort of benches or their quality of inviting resting and chatting), and

- memories (e.g., nostalgia, discrepancy).

Each of these seven sense of place categories contained participants' qualitative expressions that were rated for their valence. The dimensions of locale (from the walkability indices based on quantitative GIS analysis) and sense of place (categorized qualitative expressions rated for valence) may seem overlapping. However, they are distinguishable. For example, a shopping facility is a destination, and its availability and distance from another location is part of the locale. However, a participant's comment about using that shopping facility (desired destination with positive valence) and the difficulty of accessing the facility with limited mobility (negative valence of accessibility) conveys a sense of place as it relates the locale to personal experience and relevance. The same locale may have a different sense of place for another participant: he or she might not use that shopping facility (negative or neutral valence, if mentioned at all) or-with full mobility-may not have difficulties in accessing it. While almost all statements conveyed either a positive or negative valence, statements in the category "memories" were frequently highly emotional. This may have resulted from some participants leading the interviewer along and talking about a route of personal significance, rather than one of a daily errand.

Each expression in the study data set was assigned to one of the seven sense of place categories, and assigned a 
valence. Some expressions, such as those that include references to different times of the day, relate to more than one category or valence. For example, a park may be a preferred destination during the day (to enjoy the greenery) but may be avoided at night (for fear about safety). Rather than splitting these comments into multiple expressions for separate categorization, we kept them together and assigned them to two or more categories.

\subsection{MAPPING SENSE OF PLACE TO LOCATIONS}

To represent a feature on a map, the cartographer must first locate it. The expressions collected during the walking interviews were initially mapped to the positions along the routes where they were made. However, we also attempted to identify locations that each expression referred to. Doing so was challenging, as the spatial references could be vague or might refer to an ill-defined area, or even to a shape that varies depending on the context. For example, a comment about the benches along street $\mathrm{X}$ might refer not to the common geographic definition and length of the whole street, but only to the part of the street that offers a specific type of resting opportunity. For the MABF study data, we assigned expressions a location and an extent based on explicit references to locations when available, and made assumptions based on the content of the expression when explicit references were missing. Expressions that related to small features were mapped to a point and assigned an "uncertainty size" to reflect the area that might possibly be covered by the expression. Expressions referring to larger, identifiable features (for example, a street, a park, or the historic center of a city) we mapped to points at representative locations within or along those features, and assigned an "uncertainty size."

\section{DIMENSIONS OF AND APPROACHES TO VISUALLY-SUPPORTED DATA ANALYSIS}

To VISUALly SUPPORT QDA, different aspects of the analytical process need to be considered: the specific tasks to be completed, the data and their dimensions, and how those data dimensions map to visual variables, in order to define symbolization options.

\subsection{QDA TASKS}

QDA requires conceptualization and sense-making, and its core principles include describing or categorizing data, and making connections between categories (Dey 1993). Visual representations can support QDA by contributing "to conceptual clarification which can encourage us to think about the data in new ways" (Dey 1993, 218). When the qualitative data are spatial, maps or geovisualizations can be helpful by supporting typical spatial analysis tasks, such as evaluating the spatial distribution and extent of objects, the existence or absence of elements in locations, the closeness or distance of objects, or the density of space occupation.

QDA options are manifold. After discussion with the social scientists involved in the pilot study, we focused on four tasks as we explored options for visually supporting QDA. They are listed in order of a typical potential sequence for undertaking visually supported QDA, but may be revisited iteratively or even approached concurrently.

- Revising and refining categorization of sense of place. This could involve examining the spatial distribution of all data, certain data categories, or an individual participant's data (including areas covered/not covered by data), and accessing the original data chunks (i.e., the expressions) through the visualizations.

- Making connections and establishing relationships between categories or participants. By showing spatial overviews of categorized data, we can relate categories spatially and topically, finding both clusters and outliers, which leads us to consider the meaning and importance of those findings.

- Aggregating (filtered) data for focus and synthesis. For example, density overviews of selected data dimensions could be inspected, and decisions made about which data should be aggregated.

- Corroborating evidence by combining sense of place data through comparison with quantitative locale data. Additionally, corroboration might include comparing different places based on their similarity in one 
or two of Cresswell's (2009) place dimensions with regard to the other(s).

\subsection{MODELING THE DATA DIMENSIONS}

For interactive and visual data exploration, the data need a structure that allows querying and provides query results that can be transformed into visualizations. Creating a data model that includes the properties of tripartite place data and also supports data queries is a key prerequisite for creating a range of visualizations or interactive visual re-expressions. The data model needs to store the sense of place categorization and the associated qualitative data chunks (i.e., the expressions) as well as the spatial reference and its associated uncertainty. To model all aspects of sense of place, including emotions and memories, we also needed to include dimensions for associations, valence, and time. Thus, we assigned "origin," "valence," and "time" fields to each data element. Data chunks that belonged to more than one category, or that had multiple valences, were duplicated. Splitting was only used in cases when the contents were clearly separable and no holistic statements were broken up. The value of "self" in the origin field identified data chunks relating to the current location. Additional values denoted other locations containing expressions referring to the current location. The duplication or splitting of data chunks allowed us to assign multiple locations to one expression. This was useful in situations where different parts of a place were judged differently (e.g., a participant referred to a nice street but also lamented the uncomfortable benches along one stretch of the street). Temporal references were grouped into three broad categories ("distant past," "past," and "future") in order to avoid uncertainty and granularity issues such as those discussed for the spatial references.

The symbolization options we offer below (see 4.4) can be used to visualize other place-related emotion data, provided that those data conform to the generic data model described above. In sum, for our visualization options to be applicable, we assume that spatially-related emotion data are assigned a set of sense of place categories and that each categorized data chunk has a spatial reference. Additionally, to understand topical associations, the origin location needs to be available. Optional data for all data elements include the original data chunks, the uncertainty size assigned to the spatial location, a valence judgment, and an indication of time. To make use of the tripartite place definition, data about the locale dimension, for example walkability indices (see Bleisch and Hollenstein [2017] for a visual representation), may be used in combination with the sense of place dimension.

\subsection{EXPLORATORY DATA VISUALIZATION}

While some visualizations are aimed at communicating selected information, others are intended instead for visual exploration, allowing data experts to gain insight into the data using different kinds of reasoning (Gahegan 2005). Plaisant, Fekete, and Grinstein (2008) define an insight as a "nontrivial discovery" resulting from the data. Gaining insight and learning from the data through exploratory data visualization thus directly supports the main aims of QDA: sense-making and understanding (Dey 1993). Gahegan (2005) argues strongly for visualization approaches that put reasoning tasks in focus rather than concentrating on tools and accommodating data variations. Nevertheless, visual data exploration requires interactive rather than static views, thus tools allowing for data manipulation, re-expression, or viewpoint change are needed (Slocum et al. 2009).

All visual data exploration requires attention and a conscious effort to overcome innate human biases, such as cognitive inertia or confirmation bias, which can influence or potentially hinder visual data exploration. Hartwig and Dearing (1979) argue that exploratory data analysis requires openness and skepticism. From our experience, these characteristics are still strongly required but neither always present nor reported on. One option for supporting openness is re-expression-representing the data in more than one way-thus offering different perspectives on the data (Slocum et al. 2009; Hartwig and Dearing 1979). Those perspectives may be of varying usefulness for generating insight and stimulating hypotheses during exploratory visual analysis. However, adhering to the re-expression principle is essential, especially when experts who have already worked with the data set and want to look at it with fresh eyes are involved (Bleisch, Duckham, and Pettit 2017), as was the case here.

Exploratory visualizations can be implemented in a range of existing tools, or customized tools can be developed for a specific application area. We implemented our visualizations in QGIS, which allows the implementation of a wide range of symbolizations. It also offers opportunities to import data from various formats, a range of options for interacting with the data display (e.g., zooming and 
panning), and options for data filtering without requiring additional software development.

\subsection{MAPPING DATA DIMENSIONS TO VISUAL VARIABLES}

It is important to map data dimensions to the visual variables that most effectively represent them and that evoke the correct visual impression. In Table 1, we suggest a series of appropriate pairings of visual variables with sense of place data (categorized according to the model described above). These pairings are useful for the visual analysis of the data, especially as detailed above in 4.1. Below, we break down the choices involved in creating visual variable schemes for each data dimension.

Sense of place and valence: The seven categories, combined with the expression's valence, are mapped to a $7 \times 2$ diverging color scheme (Table 1 , rows $1-2$, left column). We chose complementary hues to represent opposite valences, to facilitate a natural interpretation of the valences. This choice is supported by recent findings on common color-affect associations (Bartram et al. 2017). Further, we designed the scheme so that similar sense of place categories were represented by similar hues. For example, the "safety" category often refers to infrastructure that is closely related to features that facilitate comfortable walking ("comfort"). Meanwhile, feeling safe is closely connected to the number of people who frequent a location, which in turn is related to the opportunity for social contact ("interaction"). In this way, the scheme's design follows a color circle: each color is similar in hue to its neighbors, as are the colors at the left and right ends of the rows. This is true for both the positive and negative valences. To allow the aggregation of several expressions, the scheme was extended to a $7 \times 4$ bivariate diverging color scheme (Table 1 , rows $1-2$, right column).

Spatial entity and extent: Given that the visual variable of size can have such an impact, careful consideration of how the spatial extent of an expression is represented is necessary (see 3.2). Larger features easily create an impression of greater importance compared to smaller features. In many cases, there was no clear argument for what size of symbol to use, especially for references to uncertain or ill-defined areas. Both large and small representations are sensible, but each may lead a reader to generate different hypotheses. To support fast re-expression, we have used points with different circle or ray sizes assigned for differently-sized spatial references in our visualization of the pilot study data (Table 1, row 3; we were inspired by Piatti, Reuschel, and Hurni [2013], who mainly used circular symbols to visualize similarly ill-defined places). While we ensure that the representation options adhere to visualization standards (e.g., Slocum et al. 2009; Munzner 2014), we did not optimize our choices to create the single best symbol for showing the data (as we would when mapping for communication purposes); instead, we produced a series of different options that help in developing different perspectives on the data.

Temporal and topical associations: "Memories" contained the most emotion data of any of the sense of place categories, often as associations. We differentiate two types of associations: temporal and topical associations. Memory expressions made during the walking interviews were generally evoked by the location that was passed, and link different times; we call them temporal associations. For example, passing a church may trigger memories of a wedding that took place in that church decades ago. Topical associations are triggered by characteristics and qualities of the present location, but refer to other locations and/or times with similar or opposite characteristics or qualities. For example, a participant might refer to the quality of the lighting in the current location, which they deem better than the lighting in the park at the end of the road. We used simple arrows with an origin and a pointer to represent topical associations linking different locations. The categorization of the content itself was represented through points at both ends of the associations, symbolized as discussed above.

Temporal data were not explicitly represented but instead queried through filtering-for example, by displaying information relating only to the past. In our case study data set, temporal associations or memories that link places through time often referred to the same place with different or even opposite valences. We found that interviewees' memories often involved positive perceptions of locations in the past, alongside ambivalent situations in the present, such as a loss of social contact because of deaths. As older people tend to shift their focus to more positive emotions (Charles and Leger 2016), this may be unsurprising. Nevertheless, it is interesting to analyze these circumstances with respect to the topic, place, and time dimensions concerned. 


\section{Aggregation Level}

Filtered (one participant or a selected category)

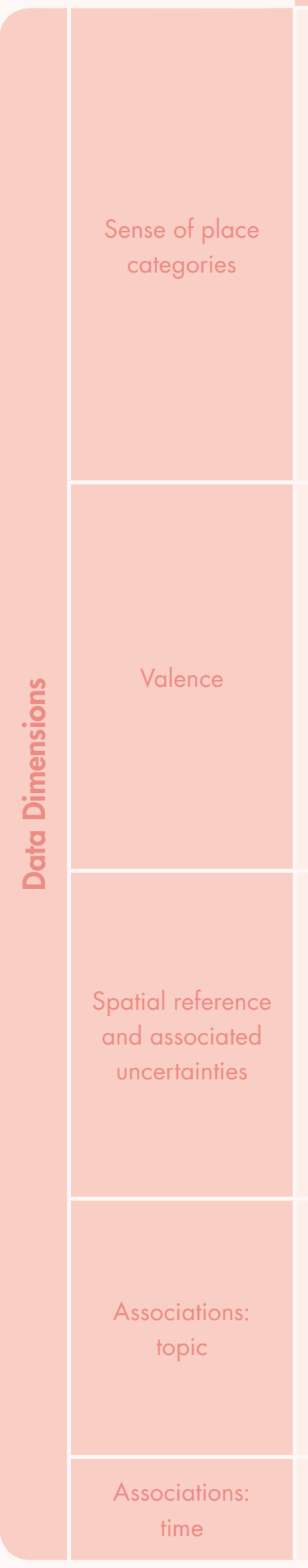

Complementary color hues

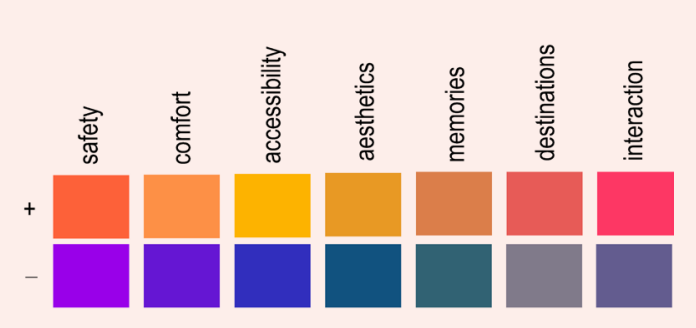

A diverging color scheme represents positive/ negative valence (Munzner 2014)
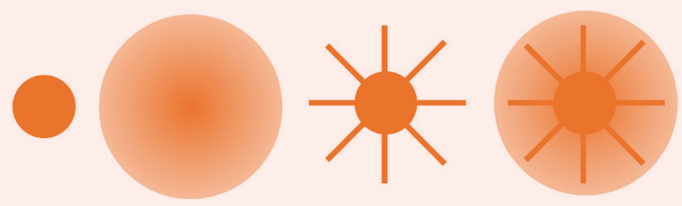

Uncertainty representation options: size and fuzziness (MacEachren et al. 2012), and radiating from a central point, inspired by Piatti, Reuschel, and Hurni (2013)
Aggregated (all participants or categories)

Semi-transparent colors; overlapping content creates more opacity

Color hues, chosen for similarity

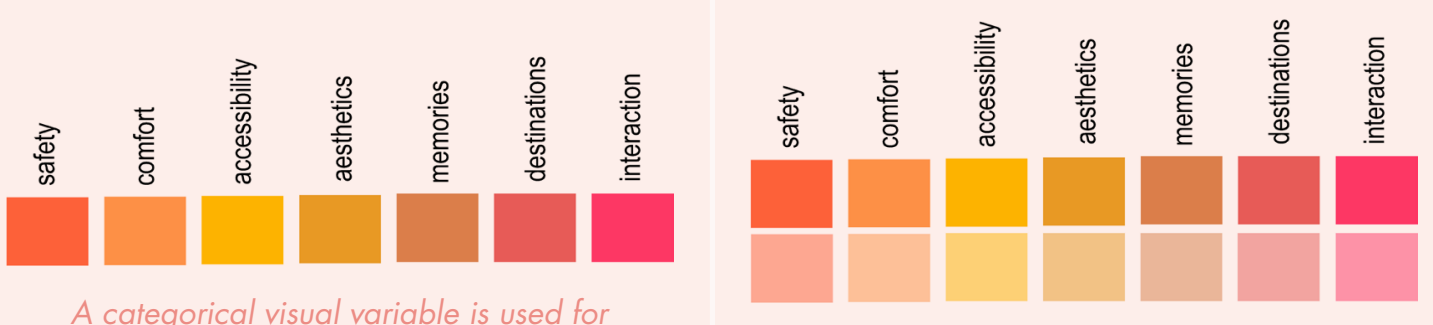

A categorical visual variable is used for categorical data (Slocum et al. 2009)

We created a bivariate color scheme by adding a numerical dimension (transparency) to the categorical dimension (Munzner 2014)

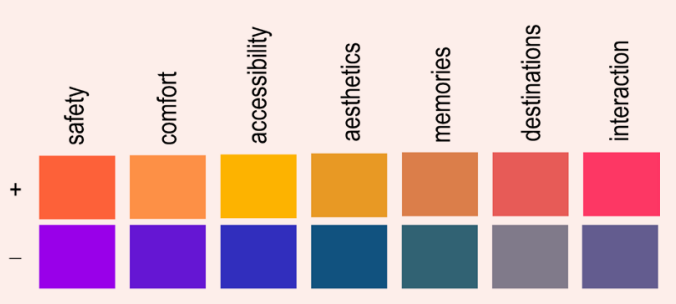

A combination of the concepts above and left, to create a bivariate diverging color scheme (Munzner 2014)

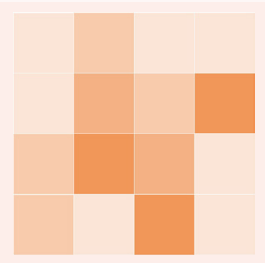

An aggregated grid view partly removes size impressions, inspired by Wood, Dykes, and Slingsby (2010)

Table 1. Overview of visual variables for the different data dimensions. Their use is illustrated in section 5. In italics, we provide the rationale for and/or references supporting choosing the respective visual variable. 
The list of visual variables and symbolization options we present here is by no means exhaustive and was condensed and selected through discussions with social scientists about the data and visualizations. They support a wide range of different visualizations, including the QDA tasks discussed in the next section. Additionally, interaction options, such as re-expression, data selection, or filtering of the listed data dimensions, can be employed through QGIS functionality. Filtering the data dimensions allows a user, for example, to focus on one or more sense of place dimensions, exclude expressions with negative valences, or display only those topical associations that refer to the past.

However when the data are eventually represented, it is important that the original data chunks are accessible through the visual representations. Accessing the original data allows the user to revise and refine the categorization as well as to validate findings and evaluate hypotheses that are formed based on the visualization.

\section{VISUALIZATIONS SUPPORTING QDA TASKS}

Based on the data structure and symbolization options described above, we created a range of visualizations for the MABF study data set and discussed them interactively in joint sessions with the social scientists to consider the visualizations' potential and limitations for supporting QDA. Interactive joint sessions spared the social scientists from the need to handle the visualization and interaction options in QGIS. The following sections discuss some example static views from our discussion sessions, including the input from social scientists. Where helpful for understanding the potential utility of the visualizations, we highlight the dataset's contents and analysis results, as well as interaction opportunities.

\subsection{REVISING AND REFINING CATEGORIZATION}

Figure 1 shows a part of an overview visualization in which all expressions are symbolized by sense of place category and valence, according to the principles detailed in section 4.4 and Table 1. Additionally, the background map is emphasized within a buffer of 20 meters around the different routes the participants walked (darker grey background map elements within the buffer area), to show the areas covered or not covered during the walking interviews. The visualization allows a first assessment of the spatial distribution and clustering of different categories, as well as positive and negative expressions. The social

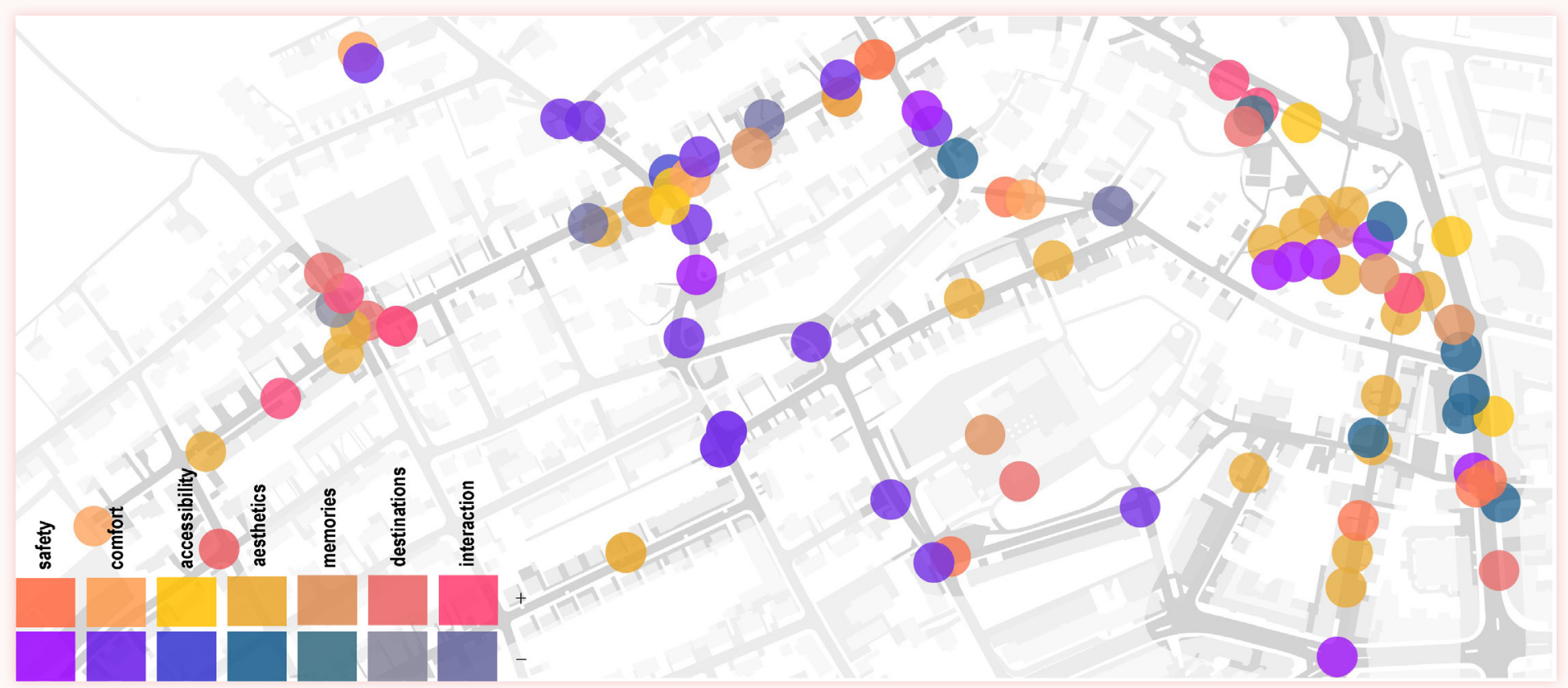

Figure 1. An extract of an overview visualization showing all localized data chunks, their categorization, and valence. The background map is subtly emphasized within a buffer of 20 meters around the routes, to show the area covered by the walking interviews. Clicking the points allows the user to view the original data chunks. 

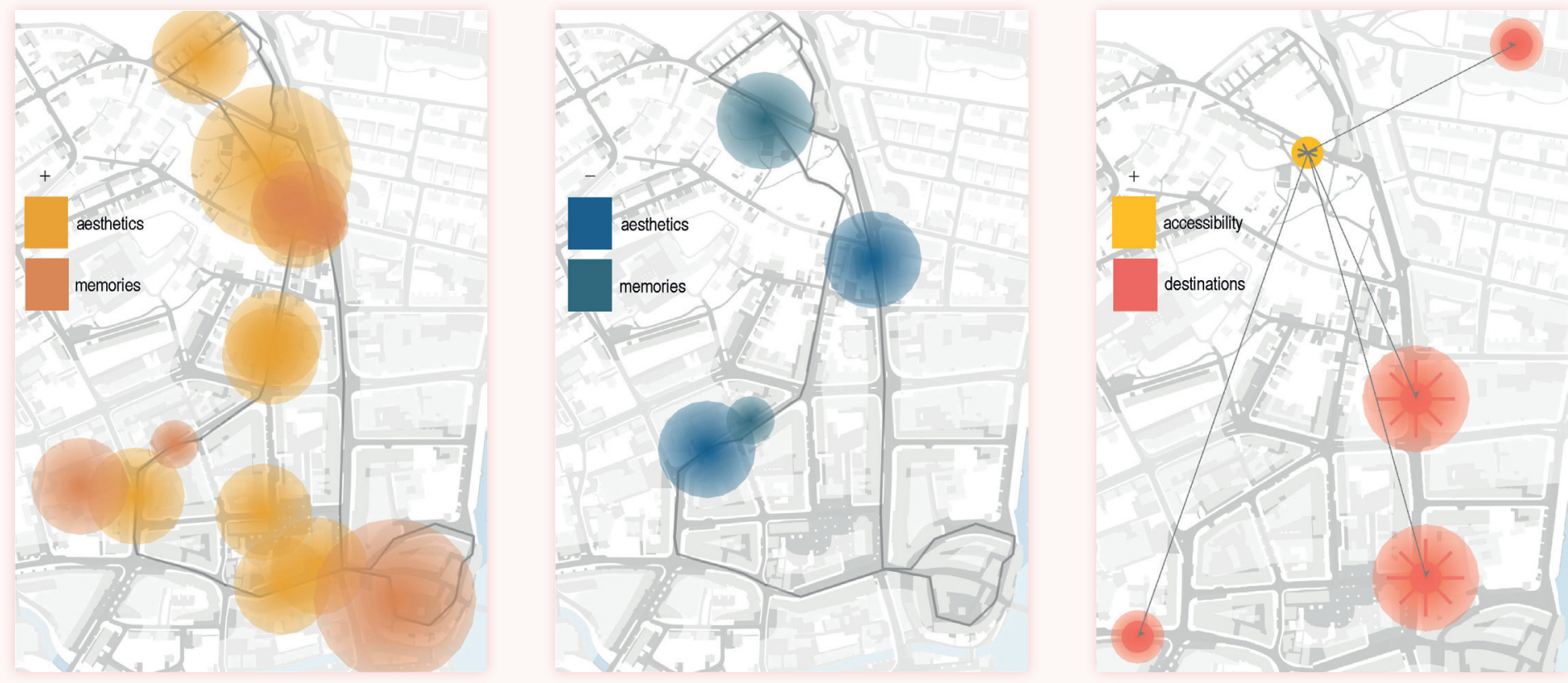

Figure 2. Filtered views of selected sense of place data. Left: Route of a single participant and their expressions of positive valence in the categories of memories and aesthetics. Circle size approximates place extent. Center: The same participant and categories as on the left, now showing negative valences. Right: Accessible destinations (red circles) referred to by a participant while standing at the location marked with a yellow circle (topical associations). The circles are used to approximate the place's location, while the overlaid opaque large dot and the rays indicate an uncertain extent associated with the location.

scientists pointed out that in QDA that does not use spatial displays, they more often think about the data semantically than spatially. The original text of the qualitative data chunks can be accessed through the point symbols to reconsider the sense of place categorization or the valence judgment, as well as to get a more detailed impression of the spatial distribution and of distance between specific statements and associations.

\subsection{MAKING CONNECTIONS AND ESTABLISHING RELATIONSHIPS}

In addition to the simple data display in Figure 1, the example visualizations in Figure 2 show filtered views of sense of place categories and expressions from a single participant, making use of the assigned uncertainty range of the spatial reference. Such visualizations clarify where along the route data were collected and where they were not (Figure 2, left and center). Caution is needed here: to some degree, these visualizations imply that locations with expressions are more important than locations that were not commented on. Likewise, the distribution of the locations, as well as the varying sizes of the circles (approximating the dimension of the mentioned places) give an impression of varying density and importance that may not accurately reflect the significance of the expressions. Using the principle of re-expression, we can vary the extent assigned to a participant's expression, or how that extent is symbolized, in order to provide varying perspectives on the data for hypothesis generation.

Discussing the filtered views, we found that looking at the expressions in the form of symbols on a map offers a different perspective on their spatiality than thinking about space only implicitly and sporadically when qualitatively analyzing text data. Specifically, Figure 2, right, revealed that the destinations (shown in red, connected by grey arrows), which the participant referred to as being within close reach, are actually located at a substantial distance from the position where the expression was made (marked with a yellow circle). Another discussion related to the negative associations of a seemingly nice area with a pond. Accessing the original expressions, we learn that while the area is nice, there are no benches upon which to rest and enjoy the view - a joyful aspect commented upon with regret. The color schemes that included very similar hues worked well in our discussion group, but one researcher 
could not see all the differences between the chosen color hues.

\subsection{AGGREGATING (FILTERED) DATA FOR FOCUS AND SYNTHESIS}

Aggregated views potentially allow the visual analysis of all data in one display. However, it is often more useful not to look at all data at once. For example, aggregation of all participants' expressions of just one category allows focusing, while looking at aggregations of several selected categories side by side may allow synthesizing findings across categories. Generally, aggregated visualizations support the same spatial analysis tasks as other representations, for example, analyzing spatial coverage and extent. With gridded aggregated visualizations (Figure 3, left), it becomes even more important to consider context information. For example, an empty area in the visualization means no data. However, there might be no data either because the area is not interesting or because a participant did not make reference to it. This is true for displays of data from single participants, but needs even more attention when data from several participants are combined.

Being able to crosscheck findings and hypotheses with the original expressions is also crucial for aggregated views. Grid-based aggregated visualizations, such as the one shown in Figure 3, left, have the advantage of not implying that the place size corresponds exactly to the area covered by the grid cells. On the other hand, the spatial extent initially assigned to the place directly influences the number of grid cells that are filled. For grid-based aggregated views, more opaque fills are created by multiple expressions that refer to the same location or overlapping place extents. Visual aggregation of expressions is achieved through the symbolization of the associated spatial uncertainty of locations with semi-transparent circles or stars in point-based displays (Figure 3, right). Importantly, interactively discussing visualizations based on different area and uncertainty assignments (employing the concept of re-expression) did stimulate different views on the data and the generation of different hypotheses. For larger areas or for larger spatial entities (for example a park or the city center), or for the identification of "hot spots," we found that grid-based aggregation of uncertainty sizes and suitably large grid cells better support topical and quantitative comparisons - a general impression of a shared sense of place-than the point-based visual aggregations of uncertainty sizes. Point based visual aggregations, especially with smaller symbol sizes, leave the single expressions discernible and support, for example, the typification of participants such as "open," "mobile," or "anxious."

\subsection{CORROBORATING EVIDENCE BY COMBINING AND EXTENDING SENSE OF PLACE WITH LOCALE}

Figure 4 shows a visualization of sense of place valence in combination with attributes of the locale. The background shows the valence of all participants' expressions that fell into the "aesthetics" sense of place category, aggregated using a grid. The grid size can be varied, but should allow
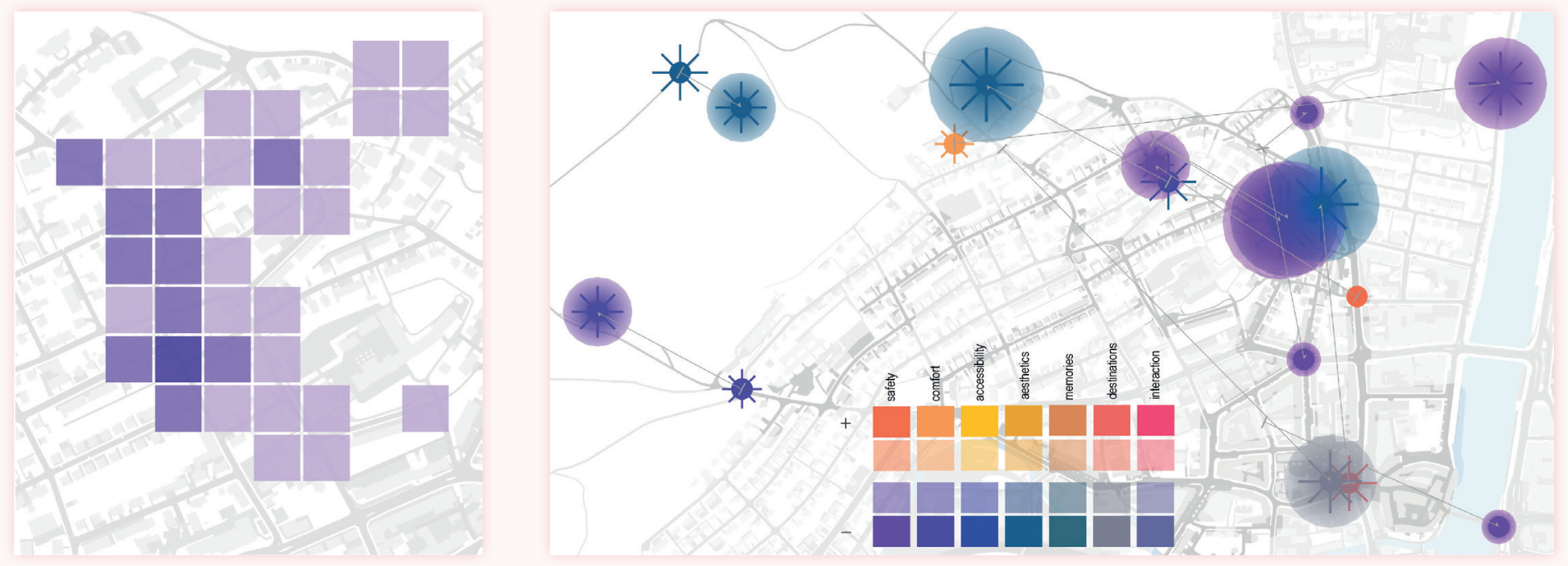

Figure 3. Overviews of selected data aggregated across all participants. Left: Grid-based aggregation of negative valence in the category "comfort" from expressions of all participants. Right: Topical associations with a negative valence, across all participants. Note: the place of origin of the association may be perceived positively. 

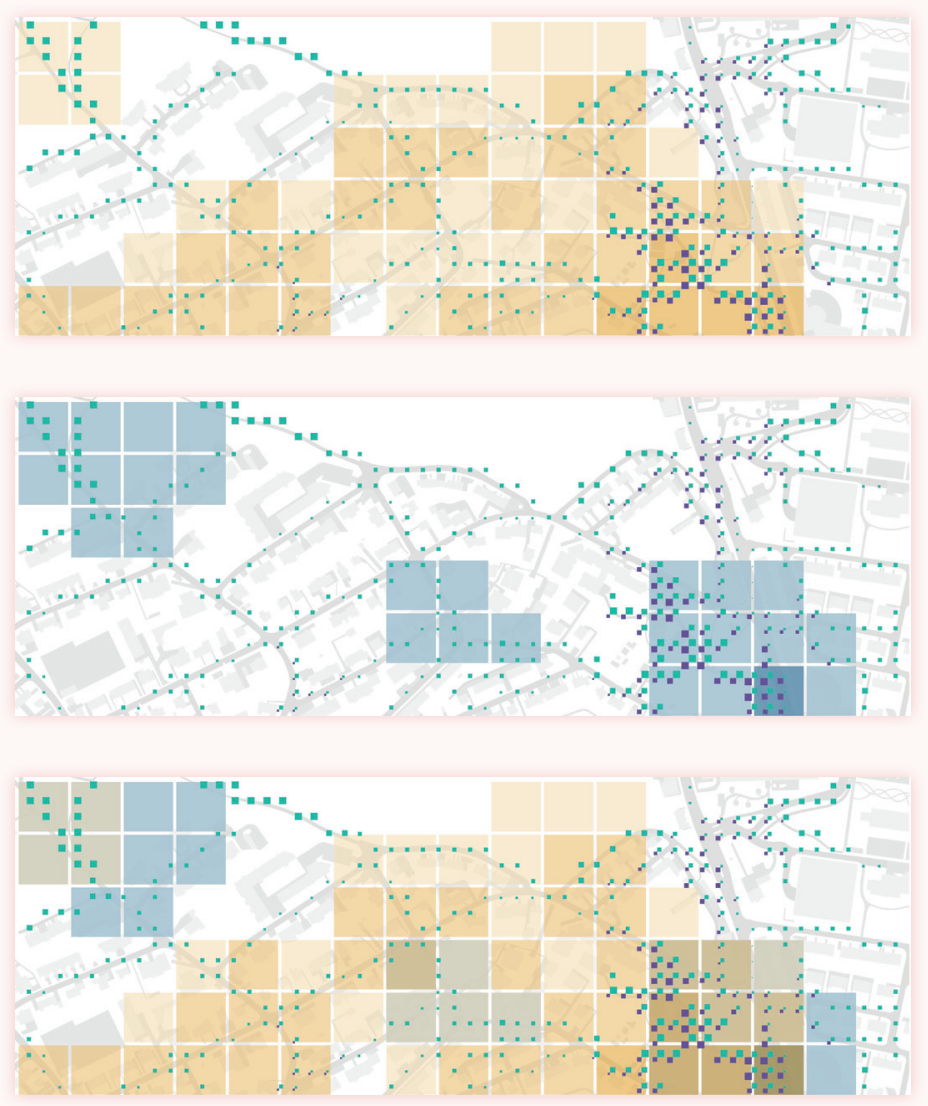

Figure 4. Gridded density overviews of the category "aesthetics," overlaid with bivariate squares symbolizing the green space index (green) and bench index (purple), calculated for short road segments (Bleisch and Hollenstein 2017). The background features expressions with a positive valence (top), negative valence (middle), or both valences (bottom).

for some aggregation of the data. The top, middle, and bottom of the figure show, in the background, aggregations of positive, negative, or both valences, respectively. While the bottom one mixes colors, this could be avoided by coloring the grid cells using the majority valence. Green space (green) and bench availability (purple) indices that were calculated along short road segments (data about the locale) are overlaid on the grids. Bigger squares indicate higher index values: i.e., more greenery or higher bench density around the road segment (see Bleisch and Hollenstein [2017] for more detail on the index visualizations). This allows the direct visual comparison of sense of place with the locale. As expected, green space is not the only contributing factor to aesthetic appeal. In the upper left corner of each of the visualizations in Figure 4, we find high green space index values, but aesthetics expressions have a predominately negative valence because of unappealing buildings in the area. Additionally, we need to consider that while the green space index is calculated along all roads, sense of place data are only available for locations that were visited or commented on. The use of the visualizations to generate hypotheses requires knowledge about the data collection procedures and evidence verification using the original data chunks.

Discussing these combined visualizations with social scientists, we find that data-dense displays are challenging, but valuable once they are understood. There is great potential to combine different aspects of the datasets and visually analyze them concurrently. In another example, pathway slope was combined with shop accessibility. The potential closing of a local shop resulted in not only some expressions discussing the loss of social contact and longer routes, but also showed concretely the much longer and steeper routes required to access alternative shopping facilities. Contrasting location characteristics with emotion data allows for a more holistic view of the affordances of an environment.

\section{CONCLUSIONS AND OUTLOOK}

WE HAVE EXPLORED how visual representations can be used to support selected tasks in the qualitative analysis of place-related emotion data. We identified symbolization opportunities for all modeled data dimensions, and exemplified them using different visualizations created with an example data set from an existing case study. We discussed these visualizations with social scientists from the original study to include their perspectives and to benefit from their data analysis knowledge. We reported insights from these discussions that we think are interesting and important for the future implementation and evaluation of the proposed visualizations for place-related emotion data in different application areas.

To conclude, we identify three areas, below, to which the findings of this study contribute. The first is that it provides specific examples of how place-related emotion data can be visualized to support QDA tasks. The second is that it makes explicit some of the benefits of the described symbolization options and how they were used in visualizations: re-expression; combinations of sense of place and locale; and visualizations of vagueness as well as topical 
associations through arrows. Finally, it advocates for visualization-supported data analysis in interdisciplinary teams.

The aspects discussed in this paper, the tripartite place definition (Cresswell 2009), the derived sense of place categories for the example data, and their inclusion in a data model, as well as the visualization options supporting selected QDA tasks and the resulting visualization, all support the analysis of emotion data related to place. While traditional QDA often only semantically considers spatial aspects of data, the options discussed here emphasize the spatial analysis of emotion data. Specifically, we find that the visualizations combining the categorized and valence-rated sense of place expressions with the locale at one location or across an area are useful for a more holistic understanding of emotion data related to place. While the individual researcher often has good knowledge of the study area to relate the collected emotion data to it, the systematic and comprehensive visual comparison of sense of place with locale was interesting and will be used more in future projects.

\subsection{SUPPORTING QDA}

Qualitative analysis is generally time consuming, and adding another exploratory method for hypothesis generation, hypothesis testing, and triangulation may only be worth the time required when significantly more or different findings are to be expected. By presenting specific examples that operationalize a generic data model for place-related emotion data based on Cresswell's (2009) tripartite place definition (section 4.2), the associated symbolization options (section 4.4), and their implementation in example visualizations, we hope to demonstrate the value of the effort required to add spatial representations to an analysis. We believe this approach should be generally applicable to data generated through walking interviews. Our discussions with our social scientist collaborators showed that the map-based visualizations, specifically the range of different visualizations offered through re-expression, provided different perspectives and triggered spatial thinking and insights. Additionally, working with the different visualizations, the social scientists found that looking at the data in a more abstract but spatially organized way-in the form of symbols on a map-offered them a different perspective on the spatiality of the data than thinking about space only implicitly and sporadically when qualitatively analyzing text data.

\subsection{SELECTED VISUALIZATION BENEFITS}

Re-expression is a basic concept in geovisualization (Slocum et al. 2009). However, it is our impression that it is rarely used extensively. One reason for this may be the time and effort needed to do so. With the generic data model and associated symbolization options, we provide a range of options that encourage active re-expression, specifically through their simplicity. In our approach, the range of visualization options is limited to point-based object representations. However, after working with the visualizations, we believe that the benefits of fast and simple re-expression outweigh this limitation. When including uncertainty information through the size of points, the visualizations give different hints and inputs for preliminary hypothesis generation, which then need evaluation by either accessing the original data chunks or yet other visualization types. Varying uncertainty-thus also varying the visual impact of the symbols - as well as making associations and their distances explicit (e.g., something some distance away is mentioned as being felt to be close) were important aspects of the implemented visualizations for analysis of this qualitative emotion data and hypothesis generation.

The combination of sense of place and locale in the same representation results in complex but insightful displays: they offer opportunities to combine subjective place impressions with the physical properties of the location, thus, supporting a more holistic assessment of the location. When crosschecking hypotheses generated about the emotion data, it was interesting to relate these data to the locale information. This also showed that participants may compare or relate locations to each other (e.g., here it now feels like it felt there some years ago) based on the characteristics of the locale and sense of place dimensions of the tripartite place definition. Such knowledge may be useful when comparing different locations from a personal perspective-for example, for a relocation. Additionally, comparing expressions of walking comfort to footpath slope allowed us to learn that a certain environmental characteristic (steepness) does foster certain expressions (of discomfort or contentment). However, a steep section of a footpath may not always be seen negatively. Perhaps, due 
to a participant's personal preference, it is not a reason for discomfort but rather an element of their personal fitness training.

Capturing more precise and/or consistent spatial relations and spatial extents of interview expressions would be interesting but may pose challenges. A persistent challenge (Çöltekin et al. 2017) is the relevant, but not necessarily precise, definition of the location and extent that is indicated by an expression, as well as specifying the temporal aspects of experience in more detail.

\subsection{INTERDISCIPLINARY ANALYSIS SETTINGS}

Discussions with colleagues in the social sciences helped us to better understand their concerns, a task which is crucial but often time-constrained in interdisciplinary research projects. We and the social scientists both benefitted greatly from working together. While QDA is generally time consuming, social scientists did not need to spend extra time on learning to handle an additional method, as we handled the re-expressions. In the team, their time was spent thinking about the visualizations and visually analyzing the example data. On the other hand, we benefited from discussions and explanations concerning, for example, the context of data collection or theoretical considerations regarding data categorization. Insights from this dialogue helped to refine the underlying data model, so that it may be transferred and applied to the visualization of similar data sets from other projects. Additionally, it helped us to define which of the many visualization options we created were most useful for which QDA tasks. Thus, the discussions shaped the content and presentation of this paper, allowing us to focus on the presentation of tasks and visualizations that have most potential for further use and evaluation in other studies, as well as for transferability to other application areas.

\subsection{OUTLOOK AND FUTURE WORK}

As we did not fully analyze data using the proposed symbolizations and visualizations, we plan to do so in a future project in the same or in a related application area with similar data sets, but with potentially different categorizations of sense of place as relevant to the application. The ideas and conclusions presented here will benefit from a more thorough evaluation and testing of the transferability of the visualization approach, an identified research area (Griffin et al. 2017). Additionally, as presented here, the data model, symbolizations, and visualizations provide some options for supporting the analysis of qualitative data, but they are by no means complete or exhaustive. To supplement the symbolization options, we suggest that abstract symbols - potentially better understood than color hues - could be added. They could be combined with the color hues or be used by themselves.

While the spatial reference or extent of emotion data seems vague, it is often the case that the expression itself is vague and/or of varying complexity. Adding more symbolization options for representing vagueness or uncertainty would further broaden the set of options for re-expression. Just as re-expression using different symbols for spatial uncertainty seemed to trigger reconsideration of data and relationships in our discussions with the social scientists, showing vagueness on the part of the participant might be worth considering in more detail in future work.

\section{ACKNOWLEDGEMENTS}

This RESEARCH USES DATA from a project funded within the FHNW (University of Applied Sciences and Arts of Northwestern Switzerland) strategic initiative "Ageing Society" but was itself not financially supported. We would specifically like to thank the social scientists of the MABF project for discussing with us the ideas presented here. Additionally, we thank the participants of the $\mathrm{MABF}$ project for sharing their thoughts and emotions about their environment. Their openness and participation has made this work possible in the first place. The background map data used in the figures are available from the Office of Geoinformation (Amt für Geoinformation) of the canton of Solothurn: geoweb.so.ch/geodaten/ datenbeschreibung.php?id=400427. The data we used were downloaded February 18, 2016. 
Bachmann, Nicole, Simon Süsstrunk, Susanne Bleisch, Daria Hollenstein, Carlo Fabian, Timo Huber, Sandra Janett, Darjan Hil, and Janine Jäger. 2016. Abschlussbericht Projekt 'Mit Den Augen Betagter Frauen, $M A B F$ ' Project Report, Strategic Initiative 'Ageing Society.' Olten, Switzerland: FHNW (University of Applied Sciences and Arts of Northwestern Switzerland).

Barclay, Sheena. 2013. “The Meaning of Cartography: Perspectives from Collins Bartholomew and the Times Atlas." The Cartographic Journal 50 (2): 121-127. doi: 10. 1179/0008704113Z.00000000077.

Bartram, Lyn, Patra Abhisekh, and Maureen Stone. 2017. “Affective Color in Visualization." In Proceedings of the 2017 CHI Conference on Human Factors in Computing Systems, 1364-1374. New York: ACM. doi: 10.1145/3025453.3026041.

Bennett, Erica V., Laura Hurd Clarke, Kent C. Kowalski, and Peter R.E. Crocker. 2017. "From Pleasure and Pride to the Fear of Decline: Exploring the Emotions in Older Women's Physical Activity Narratives." Psychology of Sport and Exercise 33: 113-122. doi: 10.1016/j.psychsport.2017.08.012.

Berrios, Raul, Peter Totterdell, and Stephen Kellett. 2018. "When Feeling Mixed Can Be Meaningful: The Relation Between Mixed Emotions and Eudaimonic Well-Being." Journal of Happiness Studies 19 (3): 841861. doi: 10.1007/s10902-017-9849-y.

Bleisch, Susanne, Matt Duckham, and Chris Pettit. 2017. "The Influence of Domain Expertise on Visual Overviews of Spatiotemporal Data." International Journal of Cartography 3 (2): 166-186. doi: 10.1080/23729333.2017.1294820.

Bleisch, Susanne, and Daria Hollenstein. 2017. "Exploring Multivariate Representations of Indices along Linear Geographic Features.” In ICC 2017: Proceedings of the 2017 International Cartographic Conference. Washington, DC: International Cartographic Association.
Carpiano, Richard M. 2009. "Come Take a Walk with Me: The 'Go-Along' interview as a Novel Method for Studying the Implications of Place for Health and Well-Being." Health and Place 15 (1): 263-272. doi: 10.1016/j.healthplace.2008.05.003.

Charles, Susan T., and Kate A. Leger. 2016. "Age and Emotion." In Encyclopedia of Mental Health, Second Edition, edited by Howard S. Friedman, 29-32. Oxford: Academic Press. doi: 10.1016/ B978-0-12-397045-9.00104-X.

Çöltekin, Arzu, Susanne Bleisch, Gennady Andrienko, and Jason Dykes. 2017. "Persistent Challenges in Geovisualization - A Community Perspective." International Journal of Cartography 3 (1): 1-25. doi: 10.1080/23729333.2017.1302910.

Coppin, Géraldine and David Sander. 2016. "Theoretical Approaches to Emotion and Its Measurement." In Emotion Measurement, edited by Herbert L. Meiselman, 3-30. Duxford, UK: Woodhead Publishing.

Couclelis, Helen. 2003. "The Certainty of Uncertainty: GIS and the Limits of Geographic Knowledge." Transactions in GIS 7 (2): 165-175. doi: 10.1111/1467-9671.00138.

Cresswell, Tim. 2009. "Place.” In International Encyclopedia of Human Geography, edited by Rob Kitchin and Nigel Thrift, 169-177. Oxford: Elsevier.

Deutsch, Kathleen, Seo Youn Yoon, and Konstadinos Goulias. 2013. "Modeling Travel Behavior and Sense of Place Using a Structural Equation Model.” Journal of Transport Geography 28: 155-163. doi: 10.1016/j. jtrangeo.2012.12.001.

Dey, Ian. 1993. Qualitative Data Analysis - A UserFriendly Guide for Social Scientists. London and New York: Routledge. doi: 10.1136/ebnurs.2011.100352. 
Ekman, Paul. 1984. "Expression and the Nature of Emotion." In Approaches to Emotion, edited by Klaus R. Scherer and Paul Ekman, 319-343. Hillsdale, NJ: Lawrence Erlbaum.

Fehr, Beverley, and James A. Russell. 1984. "Concept of Emotion Viewed From a Prototype Perspective." Journal of Experimental Psychology: General 113 (3): 464-486.

Ferreira, Inês A., Maria Johansson, Catharina Sternudd, and Ferdinando Fornara. 2016. "Transport Walking in Urban Neighbourhoods-Impact of Perceived Neighbourhood Qualities and Emotional Relationship." Landscape and Urban Planning 150: 60-69. doi: 10.1016/j.landurbplan.2016.02.009.

Gahegan, Mark. 2005. "Beyond Tools: Visual Support for the Entire Process of GIScience.” In Exploring Geovisualization, edited by Jason Dykes, Alan M. MacEachren, and Menno-Jan Kraak, 83-99. Amsterdam: Elsevier.

Gebhard, Ulrich, and Thomas Kistemann. 2016.

Landschaft, Identität Und Gesundheit - Zum Konzept Der Therapeutischen Landschaften. Wiesbaden, Germany: Springer VS. doi: 10.1007/978-3-531-19723-4.

Griffin, Amy L., Travis White, Carolyn Fish, Beate Tomio, Haosheng Huang, Claudia Robbi Sluter, João Vitor Meza Bravo, Sara I. Fabrikant, Susanne Bleisch, Melissa Yamada, and Péricles Picanço. 2017. "Designing Across Map Use Contexts: A Research Agenda." International Journal of Cartography, 3 (1): 90-114. doi: 10.1080/23729333.2017.1315988.

Griffin, Amy L., and Julia McQuoid. 2012. "At the Intersection of Maps and Emotion: The Challenge of Spatially Representing Experience." Kartographische Nachrichten 62 (6): 291-299.

Han, Ke-Tsung. 2017. “The Effect of Nature and Physical Activity on Emotions and Attention While Engaging in Green Exercise." Urban Forestry and Urban Greening 24: 5-13. doi: 10.1016/j.ufug.2017.03.012.

Hartwig, Frederick, and Brian E. Dearing. 1979. Exploratory Data Analysis. Newbury Park, CA: Sage Publications.
Hayden, Kathleen M., Bruce R. Reed, Jennifer J. Manly, Douglas Tommet, Robert H. Pietrzak, Gordon J. Chelune, Frances M. Yang, Andrew J. Revell, David A. Bennett, and Richard N. Jones. 2011. "Cognitive Decline in the Elderly: An Analysis of Population Heterogeneity." Age and Ageing 40 (6): 684-689. doi: 10.1093/ageing/afr101.

Hollenstein, Daria and Susanne Bleisch. 2016.

"Walkability for Different Urban Granularities."

International Archives of the Photogrammetry, Remote Sensing and Spatial Information Sciences 41 (July): 703708. doi: 10.5194/isprsarchives-XLI-B2-703-2016.

Iglesias, Carlos A., J. Fernando Sanchez-Rada, Gabriela Vulcu, and Paul Buitelaar. 2017. "Linked Data Models for Sentiment and Emotion Analysis in Social Networks." In Sentiment Analysis in Social Networks, edited by Federico Alberto Pozzi, Elisabetta Fersini, Enza Messina, and Bing Liu, 50-69. Cambridge: Morgan Kaufmann.

Jager, Gerry. 2016. "Short-Term Time Structure of Food-Related Emotions: Measuring Dynamics of Responses." In Emotion Measurement, edited by Herbert L. Meiselman, 273-298. Duxford, UK: Woodhead Publishing.

Jenkins, Andrew, Arie Croitoru, Andrew T. Crooks, and Anthony Stefanidis. 2016. "Crowdsourcing a Collective Sense of Place." PLoS ONE 11 (4): 1-20. doi: 10.1371/journal.pone.0152932.

Jones, Phil, Griff Bunce, James Evans, Hannah Gibbs, and Jane Ricketts Hein. 2008. "Exploring Space and Place With Walking Interviews." Journal of Research Practice 4 (2): 1-9.

Kinkeldey, Christoph, Alan M. MacEachren, Maria Riveiro, and Jochen Schiewe. 2017. "Evaluating the Effect of Visually Represented Geodata Uncertainty on Decision-Making: Systematic Review, Lessons Learned, and Recommendations." Cartography and Geographic Information Science 44 (1): 1-21. doi: 10.1080/15230406.2015.1089792. 
Kremer, Stefanie, and Louise den Uij1. 2016. "Studying Emotions in the Elderly." In Emotion Measurement, edited by Herbert L. Meiselman, 537-571. Duxford, UK: Woodhead Publishing.

Kuppens, Peter, and Philippe Verduyn. 2017. "Emotion Dynamics." Current Opinion in Psychology 17: 22-26. doi: 10.1016/j.copsyc.2017.06.004.

Lengen, Charis. 2016. "Places: Orte Mit Bedeutung." In Landschaft, Identität Und Gesundheit - Zum Konzept Der Therapeutischen Landschaften, edited by Ulrich Gebhard and Thomas Kistemann, 19-29. Wiesbaden, Germany: Springer Fachmedien.

MacEachren, Alan M. 1992. Visualizing Uncertain Information. Cartographic Perspectives, 13: 10-19. doi: 10.14714/CP13.1000.

MacEachren, Alan M., Robert E. Roth, James O’Brien, Bonan Li, Derek Swingley, and Mark Gahegan. 2012. "Visual Semiotics \& Uncertainty Visualization: An Empirical Study." IEEE Transactions on Visualization and Computer Graphics 18 (12): 2496-2505. doi: 10.1109/TVCG.2012.279.

Masdeval, Christian and Adriano Veloso. 2015. "Mining Citizen Emotions to Estimate the Urgency of Urban Issues." Information Systems 54: 147-155. doi: 10.1016/j. is.2015.06.008.

Mayring, Philipp. 2014. Qualitative Content Analysis: Theoretical Foundation, Basic Procedures and Software Solution. Klagenfurt, Austria: Alpen-Adria Universität.

Mohammad, Saif M. 2016. "Sentiment Analysis: Detecting Valence, Emotions, and Other Affectual States from Text." In Emotion Measurement, edited by Herbert L. Meiselman, 201-237. Duxford, UK: Woodhead Publishing.

Moschis, George P. 2003. "Marketing to Older Adults: An Updated Overview of Present Knowledge and Practice." Journal of Consumer Marketing 20 (6): 51625. doi: 10.1108/07363760310499093.

Munzner, Tamara. 2014. Visualization Analysis E Design. Boca Raton, FL: CRC Press.
Piatti, Barbara, and Lorenz Hurni. 2009. "Mapping the Ontologically Unreal - Counterfactual Spaces in Literature and Cartography." The Cartographic Journal 46 (4): 333-342. doi: 10.1179/000870409X125543509 47386.

Piatti, Barbara, Anne-Kathrin Reuschel, and Lorenz Hurni. 2013. "Dreams, Longings, Memories Visualising the Dimension of Projected Spaces in Fiction." In Proceedings of the 26th International Cartographic Conference of the ICA, edited by Manfred L. Buchroithner, 139. http://www.literaturatlas.eu/ files/2014/01/Piatti_ICC2013_final.pdf.

Plaisant, Catherine, Jean-Daniel Fekete, and Georges Grinstein. 2008. "Promoting Insight-Based Evaluation of Visualizations: From Contest to Benchmark Repository." IEEE Transactions on Visualization and Computer Graphics 14 (1): 120-134. doi: 10.1109/ tvcg.2007.70412.

Slocum, Terry A., Robert B. McMaster, Fritz C. Kessler, and Hugh H. Howard. 2009. Thematic Cartography and Geovisualization, Third Edition. Upper Saddle River, NJ: Pearson Prentice Hall.

Smith Mason, Jennifer, Alexander Klippel, Susanne Bleisch, Aidan Slingsby, and Stephanie Deitrick. 2016. "Approaching Spatial Uncertainty Visualization to Support Reasoning and Decision-Making." Spatial Cognition E Computation 16: 97-105. doi: 10.1080/13875868.2016.1138117.

Süsstrunk, Simon, Nicole Bachmann, Sandra Janett, and Carlo Fabian. 2018. "Mit den Augen betagter Frauen." Angewandte Gerontologie 4 (18): 13-16.

Tuan, Yi-Fu. 1979. "Space and Place: Humanistic Perspective." In Philosophy in Geography, edited by Stephen Gale and Gunnar Olsson, 387-427. doi: 10.1007/978-94-009-9394-5_19.

Wood, Denis. 1992. The Power of Maps. New York: Guilford Press.

Wood, Jo, Jason Dykes, and Aidan Slingsby. 2010. "Visualisation of Origins, Destinations and Flows with OD Maps." The Cartographic Journal 47 (2): 117-129. doi: 10.1179/000870410X12658023467367. 